\title{
Comparison of the Time-Delay Margin of a Distributed and Centralized Observer
}

\author{
Haik Silm ${ }^{1,2,3,4}$, Rosane Ushirobira ${ }^{3,1,2}$, Denis Efimov ${ }^{3,1,2,7}$, \\ Wim Michiels ${ }^{5}$, Jean-Pierre Richard ${ }^{1,2,3,4}$, Emilia Fridman ${ }^{6}$
}

\begin{abstract}
The benefit of a distributed observer concept for large-scale linear plants is shown by taking time-delays into account. It is asserted that a centralized observer suffers from delays in the measurement input, while a distributed structure allows to avoid them. In contrast, the network of the distributed observer induces communication delays among observer nodes. The delay margins for both observer concepts are estimated on a numerical example and compared using an eigenvalue-based frequency domain approach and an LMI based time-domain approach.
\end{abstract}

\section{INTRODUCTION}

Recently the problem of designing distributed observers and controllers has drawn a lot of attention [1] thanks to ongoing technological advancements, which have enabled the installation of computational and communication capacities everywhere, bringing us large-scale collaborative sensor and actuator networks. These augmented complex systems require new observer concepts, where the task of state estimation is distributed among a network of observer nodes, since centralized concepts may be unfeasible due to various engineering and economical obstacles. Most commonly, the distributed estimation problem appears in the situation when the aim is to achieve state-omniscience [2], [3] at the sensor/actuator level, meaning that each node is able to reconstruct the full state of the plant.

Following [4], [5], in this work we are going to analyze and motivate the distributed observer design in terms of robustness with respect to communication delays. Moreover, we compare it with a centralized observer and show that in terms of delay effects it is indeed advantageous to use a distributed observer. This is done by evaluating the amount of delays that is possible to sustain (the delay margin [6]) in both cases. The idea is that in the distributed setting, the delays affect on a different point than in the centralized case due to the exchange of state estimates with neighboring nodes only.

\footnotetext{
${ }^{1}$ Univ. Lille, UMR 9189 -CRIStAL, 59000 Lille, France

2 CNRS, UMR 9189, 59000 Lille, France

${ }^{3}$ Inria, Center Lille-Nord Europe, 59650 Villeneuve d'Ascq, France

${ }^{4}$ Centrale Lille, 59000 Lille, France

${ }^{5}$ Department of Computer Science, KU Leuven, 3001 Heverlee, Belgium

${ }^{6}$ Department of Electrical Engineering and Systems, Tel Aviv University, 69978 Tel Aviv, Israel

7 Department of Control Systems and Informatics, ITMO University, 197101 Saint Petersburg, Russia

This work has been partially supported by the project $\mathrm{UCoCoS}$, funded by the European Union's Horizon 2020 research and innovation program under the Marie Skłodowska-Curie Grant Agreement No 675080. It was also partially funded by Russian Science Foundation (Project 17-19-01422), the Government of Russian Federation (Grant 074-U01) and the Ministry of Education and Science of Russian Federation (Project 14.Z50.31.0031).
}

It is well-known that communication is not instantaneous. In this sense, the proposed distributed framework rises as a good alternative instead of a more straightforward solution that would be to transmit the measurements across the whole sensor network, with centralized observers at each node, which would induce high delays. Therefore we simultaneously show the advantage of distributed observers exchanging state estimates instead of measurements when delays are taken into account, bringing a new perspective to further research in this direction.

The evaluation is made using a frequency domain and a time-domain approach on a numerical example, since making the argument analytically does not seem conceivable. The reason why both methods are used is that the eigenvaluebased approach provides less conservative estimates on delay margins. At the same time, the time-domain approach is computationally feasible and it can be further extended to be later applied for nonlinear problems.

In summary, in Section II we will introduce the underlying centralized and distributed observer structures with the expected time-delays. Then in Section III, after formulating their error dynamics, we describe how the observer gains for an optimal delay margin can be obtained using direct optimization or LMIs. In Section IV these methods are used on a simulation example to demonstrate our proposition about the benefits of distributed observers exchanging state estimates.

The following notation is adopted to describe the observer network. We denote the underlying graph by $\mathcal{G}=(\mathcal{V}, \mathcal{E})$, with the set of nodes $\mathcal{V}=\{1, \ldots, N\}$ and edges $\mathcal{E} \subset \mathcal{V} \times \mathcal{V}$. By $\mathcal{V}_{i}=\{j:(j, i) \in \mathcal{E}\}$ we denote the set of nodes supplying information to node $i$. Its cardinality is the in-degree $p_{i}$. The out-degree of a node is denoted as $q_{i}$.

\section{PROBlem Formulation}

Let us consider the problem of observation of an autonomous LTI plant

$$
\dot{x}(t)=A x(t), \quad y_{i}(t)=C_{i} x(t), \quad t \geq 0,
$$

where $x(t) \in \mathbb{R}^{n}$ is the state of the plant, $A \in \mathbb{R}^{n \times n}$ is the dynamics matrix, $y_{i}(t) \in \mathbb{R}^{m_{i}}$ is the output of the $i^{\text {th }}$ sensor, $i=1, \ldots, N, N>0$ is the number of sensors, $m=\sum_{i=1}^{N} m_{i}$, and $C_{i} \in \mathbb{R}^{m_{i} \times n}$. The delays in a centralized and distributed observer are introduced following the structure given in Fig. 1 and Fig. 2, respectively. The delays from the plant to the sensor are disregarded as they are the same for both cases and assumed to be negligible since the sensors are located close to the plant. 


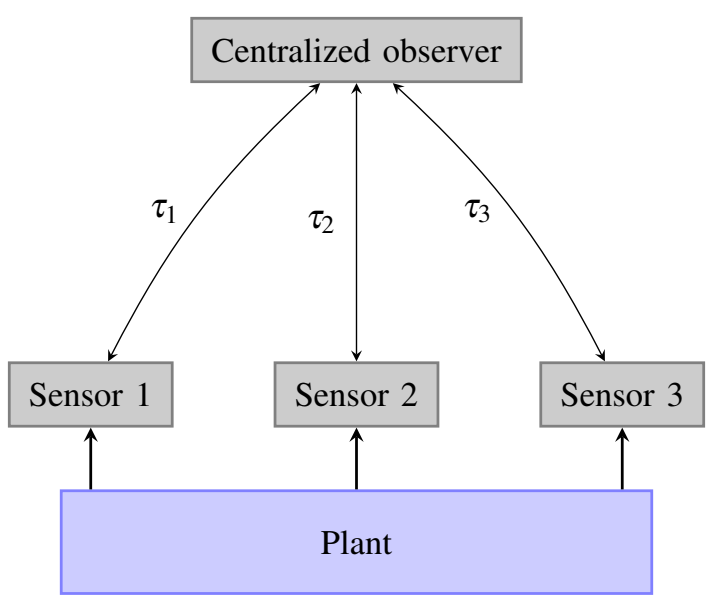

Fig. 1. Centralized observer structure

\section{A. Centralized observer}

In the centralized case, we consider a single vector output composed by all scalar outputs coming from the sensors, with the simplification that all the delays are the same $\tau_{i}=\tau$, $i=1, \ldots, N$ :

$$
y(t)=C x(t-\tau), \quad C=\operatorname{col}\left\{C_{i}\right\}_{i=1}^{N} .
$$

The corresponding Luenberger observer with state estimate $z(t) \in \mathbb{R}^{n}$ is

$$
\dot{z}(t)=A z(t)+L(C z(t-\tau)-y(t)),
$$

where $L \in \mathbb{R}^{n \times m}$ is a gain matrix to be designed [7]. Note that in Fig. 1, the presence of double transmission arrows between the nodes and the centralized observer implies that another delay takes place when the information is transmitted back to the sensors. It is not present in the equations above since it does not influence the observer stability, however, it will play a role if the generated state estimates are used for a distributed regulation.

\section{B. Distributed observer}

In the distributed setting, each sensor will act as a local observer $i$ based on its measurement, and the observer nodes are connected according to the communication graph $\mathcal{G}$. Although the pair $(A, C)$ is observable, the pair $\left(A, C_{i}\right)$ may be not. Therefore an information exchange is necessary for the distributed observer, which is selected in the form following [8]:

$$
\begin{aligned}
\dot{z}_{i}(t)=A z_{i}(t)+ & L_{i}\left(C_{i} z_{i}(t)-y_{i}(t)\right) \\
& +\sum_{j \in \mathcal{V}_{i}} H_{i, j}\left(z_{i}\left(t-\tau_{i, j}\right)-z_{j}\left(t-\tau_{i, j}\right)\right) .
\end{aligned}
$$

where a second term, called consensus term is added, according to the coupling in $\mathcal{G}$. The gains $H_{i, j} \in \mathbb{R}^{n \times n}$ and $L_{i} \in \mathbb{R}^{n \times m_{i}}$ have to be designed to maximize the delay margin $\tau_{i, j}$, which denotes the delay of information transmission from observer node $i$ to $j$. Here we assume that $H_{i, j}=0$ if the nodes $i$ and $j$ are disconnected.

Remark 1: The main assumption is that the delays are known and their variation can be neglected.

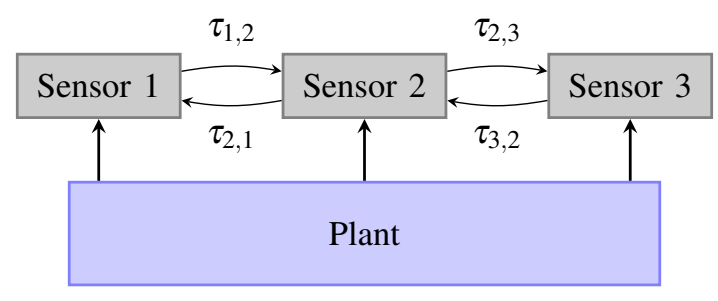

Fig. 2. Distributed observer structure

\section{Methodology}

Introducing the observer error $e(t)=z(t)-x(t)$, the error dynamics in the case of centralized estimation can be written as follows:

$$
\dot{e}(t)=A e(t)+L C e(t-\tau) .
$$

For the distributed problem, the error for each observer node is $e_{i}(t)=z_{i}(t)-x(t)$ with the corresponding dynamics:

$$
\dot{e}_{i}(t)=\left(A+L_{i} C_{i}\right) e_{i}(t)+\sum_{j \in \mathcal{V}_{i}} H_{i, j}\left(e_{i}\left(t-\tau_{i, j}\right)-e_{j}\left(t-\tau_{i, j}\right)\right)
$$

For stability investigation, we introduce the large-scale error $E(t)=\operatorname{col}\left\{e_{i}(t)\right\}_{i=1}^{N}$ and assuming $\tau_{i, j}=\tau$ as a simplification we can write

$$
\dot{E}(t)=\bar{A} E(t)+\bar{L} \bar{C} E(t)+\mathcal{H} E(t-\tau),
$$

where $\bar{A}=\operatorname{diag}\left\{A_{i}\right\}_{i=1}^{n}, \bar{C}=\operatorname{diag}\left\{C_{i}\right\}_{i=1}^{n}, \bar{L}=\operatorname{diag}\left\{L_{i}\right\}_{i=1}^{n}$ and

$$
\mathcal{H}=\left[\begin{array}{cccc}
\sum_{j \in \mathcal{V}_{1}} H_{1, j} & -H_{1,2} & \ldots & -H_{1, N} \\
& & \ddots & \\
-H_{N, 1} & \ldots & -H_{N, N-1} & \sum_{j \in \mathcal{V}_{N}} H_{N, j}
\end{array}\right] .
$$

\section{A. Direct Optimization}

For asymptotic stability of the system (4), the necessary and sufficient condition are that the generalized eigenvalues $\lambda \in \mathbb{C}$ are in the open left half-plane for a fixed constant time-delay $\tau$. We denote the spectral abscissa with

$$
c(L):=\sup \{\Re(\lambda): \operatorname{det} M(\lambda, L)=0\}
$$

where

$$
M(\lambda, L):=\lambda I_{n}-A-L C \mathrm{e}^{-\lambda \tau}
$$

is the characteristic matrix of (4). In the spectrum-based stabilization approach, we solve the optimization problem

$$
\min _{L} c(L) \text {. }
$$

This involves two steps, the calculation of the eigenvalues with the highest real-part and the determination of the corresponding gradient [9], [10]. This approach has previously been implemented in MATLAB for the design of stabilizing fixed-order controller for time-delay systems [11] and was adapted in this work to solve the static observer problem in the centralized and distributed case. For the latter the optimization problem is solved for the gains $L_{1}, \ldots, L_{N}$, $\left\{H_{i, j} \mid(i, j) \in \mathcal{E}\right\}$ and

$$
M(\lambda, \bar{L}, \mathcal{H}):=\lambda I_{n N}-\bar{A}-\bar{L} \bar{C}-\mathcal{H} e^{-\lambda \tau} .
$$


Additionally, to compute the delay margin, the error system must be stable for all delays in $[0, \tau]$. Therefore, the optimization problem is solved for a grid of delay values simultaneously where the right-most eigenvalue of all delay values determines the gradient. Since this ensures stability only for discreet values of the delay, it is later necessary to check if the spectral abscissa is indeed negative for the whole interval $[0, \tau]$. Otherwise the optimization procedure is repeated using a finer grid.

\section{B. Lyapunov-Krasovskii}

The existence of a Lyapunov-Krasovskii functional is a sufficient condition for the asymptotic stability of a timedelay system. In the case of a system with a single pointwise constant delay $\tau$, a suitable Lyapunov-Krasovskii candidate [12] is

$$
\begin{aligned}
V\left(e_{t}, \dot{e}_{t}\right)=e^{T}(t) P \dot{e}(t)+ & \int_{t-\tau}^{t} e^{T}(s) \operatorname{Se}(s) \mathrm{d} s \\
& +\tau \int_{-\tau}^{0} \int_{t+\theta}^{t} \dot{e}^{T}(s) \operatorname{Re}(s) \mathrm{d} s \theta
\end{aligned}
$$

where $e_{t} \in \mathcal{C}_{[-\tau, 0]}$ (i.e. a continuous function defined on $[-\tau, 0])$ is the state of the system and $P, R$ and $S$ are positive definite matrices. Taking the derivative of (9) and using the descriptor method for (4) with slack variables $P_{2}, P_{3}$ [12] leads to

$$
\begin{gathered}
\dot{V}=2 e^{T}(t) P \dot{e}(t)+2\left(e^{T}(t) P_{2}^{T}+\dot{e}^{T}(t) P_{3}^{T}\right)(A e(t)+L C e(t-\tau)-\dot{e}(t)) \\
+e^{T}(t) \operatorname{Se}(t)+\tau^{2} \dot{e}^{T}(t) R \dot{e}(t)-e^{T}(t-\tau) S e(t-\tau)-\tau \int_{t-\tau}^{t} \dot{e}^{T}(s) R \dot{e}(s) \mathrm{d} s
\end{gathered}
$$

for positive definite matrices $P_{i}, S_{i}, R_{i}, W_{i}$ and matrices $X_{i}$, If $P_{3}=\varepsilon P_{2}, \varepsilon>0$ and $Y=P_{2}^{T} L$, we see that the error system is asymptotically stable for a delay $\tau$ if the LMI

$$
\left[\begin{array}{ccc}
A^{T} P_{2}+P_{2}^{T}+S-R & P-P_{2}^{T}+\varepsilon A^{T} P_{2} & Y C+R \\
* & -\varepsilon P_{2}-\varepsilon P_{2}^{T}+\tau^{2} R & \varepsilon Y C \\
* & * & -S-R
\end{array}\right]<0
$$

is feasible. Due to the convexity this is also true for any delay between zero and $\tau$ [13].

Correspondingly in the distributed case, (9) with (6) would lead to

$$
\left[\begin{array}{ccc}
\Psi & P-P_{2}^{T}+\varepsilon \bar{C}^{T} Y_{1}^{T}+\varepsilon \bar{A}^{T} P_{2} & Y_{2}+R \\
* & -\varepsilon P_{2}-\varepsilon P_{2}^{T}+\tau^{2} R & \varepsilon Y_{2} \\
* & * & -S-R
\end{array}\right]<0
$$

where $Y_{1}=P_{2}^{T} L$ and $Y_{2}=P_{2}^{T} \mathcal{H}$ and $\Psi=\bar{A}^{T} P_{2}+P_{2}^{T} \bar{A}+$ $\bar{C}^{T} Y_{1}^{T}+Y_{1} \bar{C}+S-R$. We encounter now the problem, that $\mathcal{H}$ is algebraically constrained according to (7) in a way, which cannot be enforced with $Y_{2}$ and $P_{2}$.

\section{LMI for the distributed observer}

Inspired by [14] we employ for every observer node $i=$ $1, \ldots, N$ the Lyapunov-Krasovskii functional

$$
\begin{aligned}
V_{i}\left(e_{i, t}, \dot{e}_{i, t}\right) & =e_{i}^{T}(t) P_{i} e_{i}(t)+\int_{t-\tau}^{t} \mathrm{e}^{-2 \alpha_{i}(t-s)} e_{i}^{T}(s) S_{i} e_{i}(s) \mathrm{d} s \\
+ & \tau \int_{t-\tau}^{t}(\tau+s-t) \mathrm{e}^{-2 \alpha_{i}(t-s)} \dot{e}_{i}^{T}(s) R_{i} \dot{e}_{i}(s) \mathrm{d} s,
\end{aligned}
$$

where compared to (9) there are additional terms with $\alpha_{i}>0$. A modified version of [14, Theorem 1] is necessary:

Theorem 2: Suppose there exist gains $L_{i}, \quad \tilde{H}_{i}=$ row $\left\{H_{i, j}\right\}_{j \in \mathcal{V}_{i}}$, positive definite matrices $W_{i}$ and constants $\alpha_{i}>0,0<\pi_{i}<2 \alpha_{i} q_{i}^{-1}$, such that the following vector dissipation inequality holds for all $i=1, \ldots, N$ :

$$
\begin{aligned}
\dot{V}_{i}+2 \alpha_{i} V_{i}-\sum_{j \in \mathcal{V}_{i}} \pi_{j} V_{j} & -\sum_{j \in \mathcal{V}_{i}} \mathcal{W}_{j}\left(e_{j}(t), e_{j}(t-\tau)\right) \\
& +\left(\sum_{j: i \in \mathcal{V}_{j}} \tau_{j}^{2}\right) \dot{e}_{i}^{T}(t) W_{i} \dot{e}_{i}(t) \leq 0,
\end{aligned}
$$

where $\mathcal{W}_{i}(u, z)=\frac{\pi^{2}}{4}[u-z]^{T} W_{i}[u-z]$. Then the observer network (3) achieves state-omniscience, i.e.

$$
\lim _{t \rightarrow \infty}\left\|z_{i}(t)-x(t)\right\|=0, \quad i=1, \ldots, N .
$$

A system of LMIs which ensures this relation can be derived again using the descriptor method, so that

$$
\left[\begin{array}{cccccc}
\Xi_{i, 11} & \Xi_{i, 12} & 0 & \Xi_{i, 14} & 0 & \Xi_{i, 16} \\
* & \Xi_{i, 22} & -\Psi_{i, 12} & \Xi_{i, 24} & 0 & \Xi_{i, 26} \\
0 & * & -\Psi_{i, 22} & 0 & 0 & 0 \\
* & * & 0 & \Xi_{i, 44} & 0 & \Xi_{i, 46} \\
0 & 0 & 0 & 0 & -\Phi_{i, 11} & -\Phi_{i, 12} \\
* & * & 0 & * & * & \Xi_{i, 66}
\end{array}\right]<0
$$
$U_{i}, \tilde{F}_{i}$, where

$$
\begin{aligned}
& {\left[\begin{array}{ll}
\Psi_{i, 11} & \Psi_{i, 12} \\
\Psi_{i, 21} & \Psi_{i, 22}
\end{array}\right]=\left[\begin{array}{cc}
\mathrm{e}^{-2 \alpha_{i} \tau} R_{i}-2 \alpha_{i} P_{i}^{-1}-S_{i} & -\mathrm{e}^{-2 \alpha_{i} \tau} R_{i} \\
-\mathrm{e}^{-2 \alpha_{i} \tau} R_{i} & \mathrm{e}^{-2 \alpha_{i} \tau}\left(R_{i}+S_{i}\right)
\end{array}\right] } \\
& {\left[\begin{array}{ll}
\Phi_{i, 11} & \Phi_{i, 12} \\
\Phi_{i, 21} & \Phi_{i, 22}
\end{array}\right] }=\left[\begin{array}{cc}
\operatorname{diag}\left\{\pi_{j} P_{j}+\frac{\pi^{2}}{4} W_{j}\right\}_{j \in \mathcal{V}_{i}} & -\operatorname{diag}\left\{\frac{\pi^{2}}{4} W_{j}\right\}_{j \in \mathcal{V}_{i}} \\
-\operatorname{diag}\left\{\frac{\pi^{2}}{4} W_{j}\right\}_{j \in \mathcal{V}_{i}} & \operatorname{diag}\left\{\frac{\pi^{2}}{4} W_{j}\right\}_{j \in \mathcal{V}_{i}}
\end{array}\right] \\
& \Xi_{i, 11}=\tau^{2} R_{i}+q_{i} \tau^{2} W_{i}-\varepsilon_{i} X_{i}-\varepsilon_{i} X_{i}^{T} \\
& \Xi_{i, 12}=P_{i}-X_{i}+\varepsilon_{i} X_{i}^{T} A+\varepsilon_{i} U_{i} C_{i} \\
& \Xi_{i, 14}=\varepsilon_{i} \widetilde{F}_{i}-\tilde{\varepsilon}_{i} 1_{p_{i}}^{T} \otimes X_{i} \\
& \Xi_{i, 16}=-\varepsilon_{i} \widetilde{F}_{i}-\bar{\varepsilon}_{i} 1_{p_{i}}^{T} \otimes X_{i} \\
& \Xi_{i, 22}=X_{i}^{T} A+U_{i} C_{i}+A^{T} X_{i}+C_{i}^{T} U_{i}^{T}-\Psi_{i, 11} \\
& \Xi_{i, 24}=\widetilde{F}_{i}+\tilde{\varepsilon}_{i} 1_{p_{i}}^{T} \otimes\left(A^{T} X_{i}+C_{i}^{T} U_{i}^{T}\right) \\
& \Xi_{i, 26}=-\widetilde{F}_{i}+\bar{\varepsilon}_{i} 1_{p_{i}}^{T} \otimes\left(A^{T} X_{i}+C_{i}^{T} U_{i}^{T}\right) \\
& \Xi_{i, 44}=\tilde{\varepsilon}_{i}\left(1_{p_{i}} \otimes \widetilde{F}_{i}+1_{p_{i}}^{T} \otimes \widetilde{F}_{i}^{T}\right) \\
& \Xi_{i, 46}=\bar{\varepsilon}_{i} 1_{p_{i}} \otimes \widetilde{F}_{i}-\tilde{\varepsilon}_{i} 1_{p_{i}}^{T} \widetilde{F}_{i}^{T} \\
& \Xi_{i, 66}=-\bar{\varepsilon}_{i}\left(1_{p_{i}} \otimes \widetilde{F}_{i}+1_{p_{i}}^{T} \otimes \widetilde{F}_{i}^{T}\right)-\Phi_{i, 22},
\end{aligned}
$$

has to be feasible for $i=1, \ldots, N$. Note the additional tuning variables $\varepsilon_{i}>0, \bar{\varepsilon}_{i}>0$ and $\tilde{\varepsilon}_{i}>0$ for each node. Then the gains sought-after are obtained with

$$
L_{i}=\left(X_{i}^{T}\right)^{-1} U_{i}, \quad \widetilde{H}_{i}=\left(X_{i}^{T}\right)^{-1} \widetilde{F}_{i} .
$$


Remark 3: In the special case, that the same gain $H_{i, j}=H_{i}$ is used for all neighbors $j \in \mathcal{V}_{i}$ of an observer node the LMIs can be simplified with $F_{i}=X_{i}^{T} H_{i}$ to

$$
\begin{aligned}
{\left[\begin{array}{ccccc}
\Xi_{i, 11} & \Xi_{i, 12} & \Xi_{i, 13} & 0 & \Xi_{i, 15} \\
\star & \Xi_{i, 22} & \Xi_{i, 23} & 0 & \Xi_{i, 25} \\
\star & \star & -\Psi_{i, 22} & 0 & \Xi_{i, 35} \\
0 & 0 & 0 & -\Phi_{i, 11} & -\Phi_{i, 12} \\
\star & \star & \star & \star & \Xi_{i, 55}
\end{array}\right]<0 } \\
\Xi_{i, 11}=\tau^{2} R_{i}+q_{i} \tau^{2} W_{i}-\varepsilon_{i} X_{i}-\varepsilon_{i} X_{i}^{T} \\
\Xi_{i, 12}=P_{i}-X_{i}+\varepsilon_{i} X_{i}^{T} A+\varepsilon_{i} U_{i} C_{i} \\
\Xi_{i, 13}=p_{i} \varepsilon_{i} F_{i} \\
\Xi_{i, 15}=1_{p_{i}}^{T} \otimes\left(-\bar{\varepsilon}_{i} X_{i}-\varepsilon_{i} F_{i}\right) \\
\Xi_{i, 22}=X_{i}^{T}\left(A+L_{i} C_{i}\right)+\left(A+L_{i} C_{i}\right)^{T} X_{i}-\Psi_{i, 11} \\
\Xi_{i, 23}=-\Psi_{i, 12}+p_{i} F_{i} \\
\Xi_{i, 25}=1_{p_{i}}^{T} \otimes\left(-F_{i}+\bar{\varepsilon}_{i}\left(A+L_{i} C_{i}\right)^{T} X_{i}\right) \\
\Xi_{i, 35}=p_{i} \bar{\varepsilon}_{i} 1_{p_{i}}^{T} \otimes F_{i}^{T} \\
\Xi_{i, 55}=-\bar{\varepsilon}_{i} 1_{p_{i} \times p_{i}} \otimes\left(F_{i}+F_{i}^{T}\right)-\Phi_{i, 22} .
\end{aligned}
$$

\section{EXAMPLE}

Let us demonstrate how these approaches can be used for the delay-margin comparison of centralized and distributed observers on an example. Consider a simple system

$$
A=\left[\begin{array}{cccc}
2 & 1 & -1 & -1 \\
1 & 2 & 2 & 2 \\
1 & 1 & 2 & 1 \\
-2 & -2 & 1 & 2
\end{array}\right], \quad C_{1}=\left[\begin{array}{llll}
1 & 0 & 0 & 0
\end{array}\right],
$$

in (1), thus $n=4$ and $N=2$. The system is unstable and for each output the dimension of the observable subspace is less then $n$. Thus, the system is not detectable using a single observer node.

First, we compute the delay margin for the centralized observer (2). The LMI (10) was found feasible for $\tau=0.20$, the corresponding gain is

$$
L=\left[\begin{array}{cc}
-4.9158 & -0.9099 \\
2.7431 & -7.8824 \\
0.9100 & -4.9158 \\
7.8823 & 2.7430
\end{array}\right]
$$

and $\varepsilon=2.9$. With the direct optimization method a delay margin of $\tau=0.23$ with

$$
L=\left[\begin{array}{cc}
-2.6597 & -1.2793 \\
3.1585 & -5.5244 \\
2.1407 & -5.6024 \\
4.7547 & 1.0693
\end{array}\right]
$$

was obtained. The results are illustrated in Fig. 3 and Fig. 4 with branch plots of the real-part of the right-most eigenvalues as function of the delay.

For the distributed observer both sensor nodes are connected with each other. The delay margin obtained with the

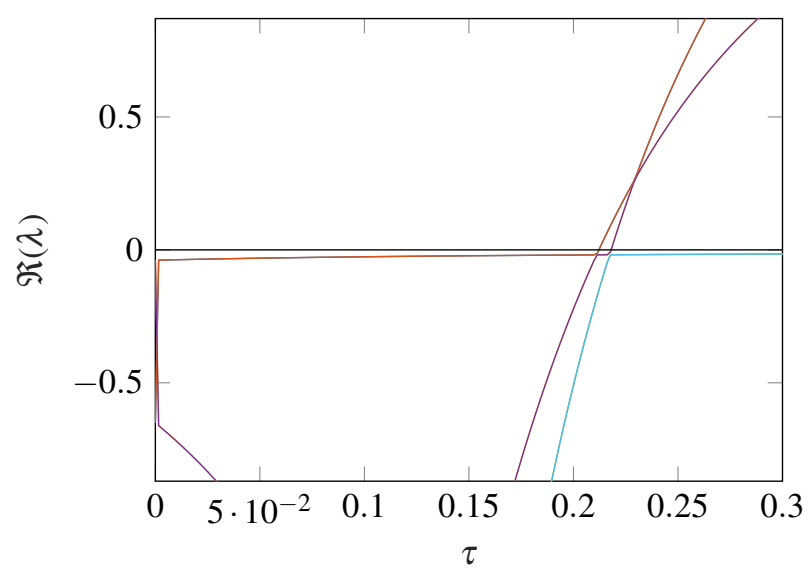

Fig. 3. Branch plot when the LMI is solved for $\tau=0.20$

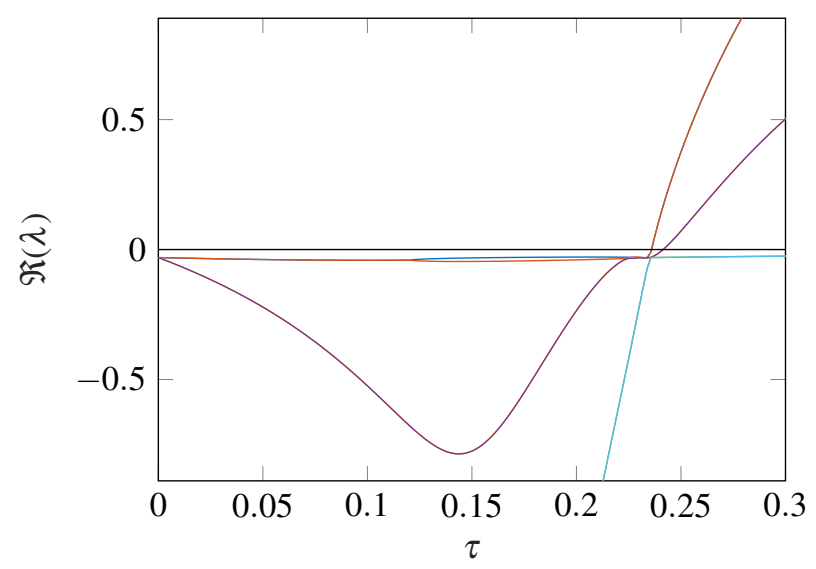

Fig. 4. Branch plot when direct optimization is used for $\tau=[0,0.23]$

direct optimization approach is $\tau=0.55$ and the gains are

$$
\begin{aligned}
& L_{1}=\left[\begin{array}{llll}
-11.7351 & -10.6626 & -3.9107 & 12.7252
\end{array}\right]^{T} \\
& L_{2}=\left[\begin{array}{lllll}
-1.4857 & -12.1517 & -4.8092 & 4.0385
\end{array}\right]^{T}
\end{aligned}
$$

and

$$
\begin{aligned}
H_{1,2} & =\left[\begin{array}{cccc}
-1.0662 & -0.4530 & -0.4304 & 1.6444 \\
-3.6936 & -0.6652 & -3.5053 & 4.3590 \\
0.7142 & 0.2199 & -3.0543 & -1.4381 \\
-0.2513 & 0.2300 & 2.0648 & -0.4715
\end{array}\right] \\
H_{2,1} & =\left[\begin{array}{cccc}
-1.1627 & 0.8724 & -0.4767 & 0.6092 \\
-0.5522 & -3.9221 & -1.4007 & 0.6231 \\
-0.7717 & -1.6703 & -1.9817 & -0.1494 \\
1.0247 & -0.6629 & -4.8756 & -2.4795
\end{array}\right] .
\end{aligned}
$$

For the distributed observer the LMI (15) was found to be feasible for $\tau=0.98$ with

$$
\begin{aligned}
& L_{1}=\left[\begin{array}{llll}
-14.9403 & -100.2453 & -56.7389 & 30.3267
\end{array}\right]^{T} \\
& L_{2}=\left[\begin{array}{llll}
53.8637 & -29.4106 & -14.8122 & -96.1801
\end{array}\right]^{T}
\end{aligned}
$$




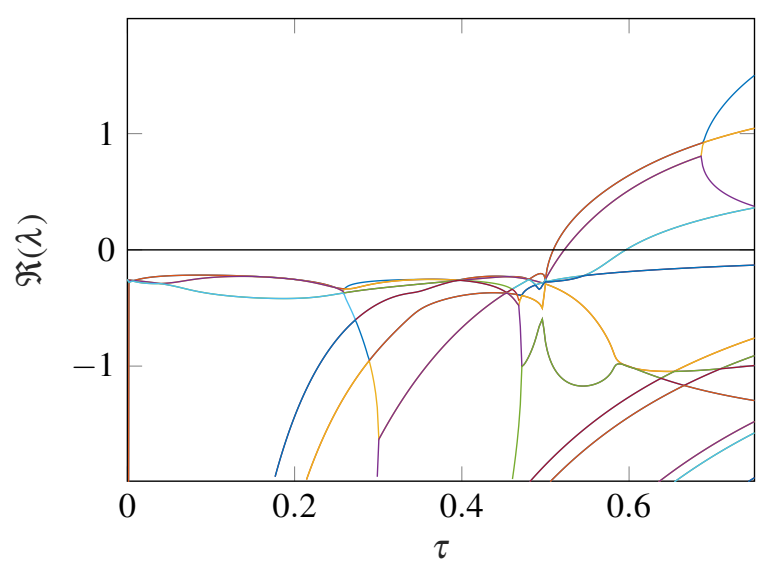

Fig. 5. Branch plot with direct optimization used for $\tau=[0,0.55]$

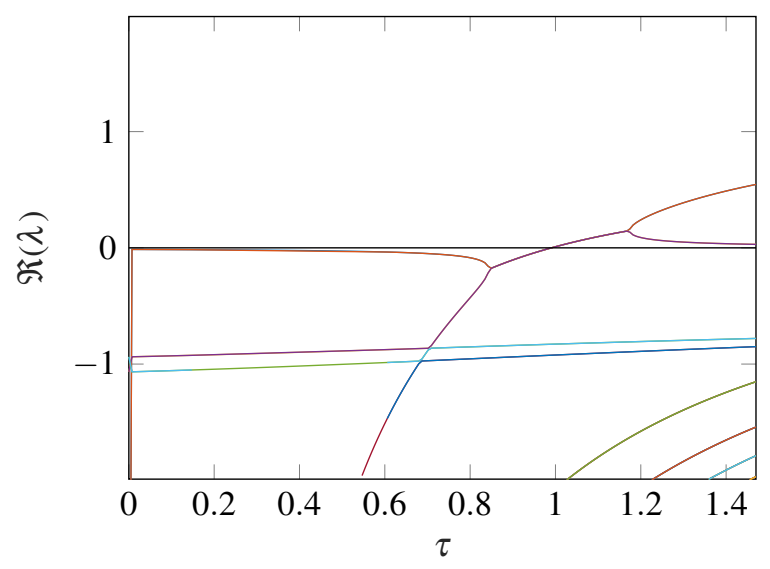

Fig. 6. Branch plot when the LMI is solved for $\tau=[0.98]$

and

$$
\begin{aligned}
H_{1,2} & =\left[\begin{array}{cccc}
-0.0081 & -0.0081 & -0.0040 & -0.0041 \\
-0.0847 & -0.0848 & -0.0404 & -0.0434 \\
0.1825 & 0.1825 & -0.8722 & 0.1400 \\
-0.2178 & -0.2178 & 0.8557 & -0.1581
\end{array}\right] \\
H_{2,1} & =\left[\begin{array}{cccc}
-0.8766 & 0.1357 & -0.1740 & -0.1740 \\
0.8607 & -0.1530 & 0.2071 & 0.2070 \\
0.0041 & 0.0043 & -0.0082 & -0.0082 \\
0.0399 & 0.0429 & -0.0818 & -0.0818
\end{array}\right]
\end{aligned}
$$

using $\varepsilon_{1}=\varepsilon_{2}=47, \bar{\varepsilon}_{1}=\bar{\varepsilon}_{2}=0$, and $\alpha_{1}=\alpha_{2}=\pi_{1}=\pi_{2}=$ 0.04. In Fig. 5 and Fig. 6 the corresponding branch plots are shown.

Bearing in mind that the LMI conditions are sufficient but not necessary, the actual margin can be higher than provided by the feasibility of the LMI. Therefore, the actual delay margins for the proposed gains can be seen in the branch plots, for which the approximate computation of eigenvalues of the direct optimization procedure were employed. The margins were additional validated by explicitly simulating the error dynamics with the obtained results.

To explicate these quantitative results, consider the observer structures in Fig. 1 and Fig. 2 with only Sensor 1 and Sensor 2. Using for example the gains (17) obtained by direct optimization of the centralized observer, the observer is only able to compensate delays which are between 0 and approximately 0.235 . If the delay exceeds this margin, the observer error will go to infinity. The distributed observer with gains (18) and (19) is able to bear communication delays up to approximately 1.0, which is higher than in the centralized case. Conversely, the delays encountered by a distributed observer are likely lower than for a centralized observer or when measurement values are transmitted through the observer network instead of exchanging state estimates.

\section{CONClusion}

Two methods for obtaining delay margins for centralized and distributed observers were presented and were used to determine an estimate of delay margins on an example. Notwithstanding that the delay margins estimated by the LMI approach are usually more conservative, both methods revealed a higher delay margin for the distributed observer. Considering that the transmission delays in a centralized concept are likely higher than the communication delay between the nodes of the distributed observer, this shows the advantage of the latter. At the same time, this serves as a justification for exchanging state estimate information between observer nodes instead of measurements, which would be prone to high delays.

\section{REFERENCES}

[1] S. Park and N. C. Martins, "Design of distributed LTI observers for state omniscience," IEEE Transactions on Automatic Control, vol. 62, no. 2, pp. 561-576, Feb. 2017.

[2] W. Han, H. L. Trentelman, Z. Wang, and Y. Shen, "A simple approach to distributed observer design for linear systems," arXiv:1708.01459 [math], Aug. 4, 2017.

[3] L. Wang and A. S. Morse, "A distributed observer for a time-invariant linear system," in 2017 American Control Conference (ACC), May 2017, pp. 2020-2025.

[4] P. Millán, L. Orihuela, C. Vivas, and F. R. Rubio, "Distributed consensus-based estimation considering network induced delays and dropouts," Automatica, vol. 48, no. 10, pp. 2726-2729, Oct. 1, 2012.

[5] P. Millán, L. Orihuela, and I. Jurado, "Delays in distributed estimation and control over communication networks," in Delays and Networked Control Systems, ser. Advances in Delays and Dynamics, Springer, Cham, 2016, pp. 199-216.

[6] R. Sipahi, "Delay-margin design for the general class of single-delay retarded-type LTI systems," International Journal of Dynamics and Control, vol. 2, no. 2, pp. 198-209, Jun. 1, 2014.

[7] H. K. Khalil, Nonlinear Systems. Prentice Hall, 2002.

[8] R. Olfati-Saber, "Distributed kalman filtering for sensor networks," in 2007 46th IEEE Conference on Decision and Control, Dec. 2007, pp. $5492-5498$.

[9] J. Vanbiervliet, K. Verheyden, W. Michiels, and S. Vandewalle, "A nonsmooth optimisation approach for the stabilisation of time-delay systems," ESAIM: Control, Optimisation and Calculus of Variations, vol. 14 , no. 3, pp. 478-493,

[10] W. Michiels, "Spectrum-based stability analysis and stabilisation of systems described by delay differential algebraic equations," IET Control Theory Applications, vol. 5, no. 16, pp. 1829-1842, Nov. 2011.

[11] W. Michiels. (2010). TDS_stabil: A MATLAB tool for fixed-order stabilization problems for time-delay systems, [Online]. Available: http: / / twr.cs. kuleuven. be/ research / software / delay-control/stab/.

[12] E. Fridman, "Tutorial on Lyapunov-based methods for time-delay systems," European Journal of Control, vol. 20, no. 6, pp. 271-283, Nov. 1, 2014.

[13] E. Fridman, Introduction to Time-Delay Systems: Analysis and Control, ser. Systems \& Control: Foundations \& Applications. Springer International Publishing, 2014. 
[14] V. Ugrinovskii and E. Fridman, "A Round-Robin type protocol for distributed estimation with $\mathscr{H}_{\infty}$ consensus," Systems \& Control Letters, vol. 69, pp. 103-110, Supplement C Jul. 1, 2014. 